\title{
Nanostructure transfer using cyclic olefin copolymer templates fabricated by thermal nanoimprint lithography
}

\author{
Arshad Khan, Shijie Li, and Xin Tang \\ Department of Mechanical Engineering, The University of Hong Kong, Pokfulam, Hong Kong, China \\ Wen-Di Li ${ }^{\mathrm{a})}$ \\ Department of Mechanical Engineering, The University of Hong Kong, Pokfulam, Hong Kong, China \\ and HKU-Shenzhen Institute of Research and Innovation (HKU-SIRI), Shenzhen 518000, China
}

(Received 29 June 2014; accepted 17 October 2014; published 29 October 2014)

\begin{abstract}
The authors demonstrate the application of cyclic olefin copolymer (COC) films as secondary nanoimprint templates for transferring sub- $100 \mathrm{~nm}$ nanostructures. Featureless COC films were first patterned by a thermal nanoimprint process using silicon molds with gratings of various periods from 140 to $420 \mathrm{~nm}$. Morphology of COC gratings imprinted at different processing parameters was characterized by scanning electron microscopy and atomic force microscopy and the grating transfer fidelity was systematically investigated. The nanoimprinted COC substrates were then used as secondary templates in an ultraviolet (UV)-cured nanoimprint lithography process to transfer the grating patterns onto UV-curable epoxy. The authors also demonstrate the application of using these nanoimprinted COC templates to transfer metallic nanostructures onto fiber facets. With good mechanical strength, high transparency to UV light, easy fabrication, and excellent chemical compatibility, COC is a promising material that can be used in low-cost secondary templates for largevolume nanoimprint-based manufacturing of nanostructured devices. (C) 2014 American Vacuum Society. [http://dx.doi.org/10.1116/1.4900609]
\end{abstract}

\section{INTRODUCTION}

Nanoimprint lithography is a promising technology that can fabricate high-resolution nanostructures beyond the limitation set by light diffraction in optical lithography or beam scattering in charged particle beam lithography. ${ }^{1}$ It has the capability to fabricate high-resolution nanostructures on large area of rigid or flexible substrates. Its unique advantages of low cost and high throughput are particularly desired by many emerging applications in the fields of flexible electronics, ${ }^{2}$ photonics, ${ }^{3}$ microfluidics, ${ }^{4}$ photovoltaics, ${ }^{5}$ just to name a few. In these emerging applications, plastic materials are widely used as device substrates mainly for their cost-effectiveness, flexibility, transparency, and lightweight, among other intriguing properties. Moreover, during the nanoimprint-based fabrication of these devices, plastic nanoimprint templates ${ }^{6}$ are also attracting increasing interest as an alternative to conventional templates made of silicon or nickel, which are more expensive and more complicatedly fabricated. The key advantage of using secondary polymer molds in production is that one does not need the master mold for every imprinting cycle therefore indirectly increases the life of the "expensive" master mold and decreases the cost of patterning. Meanwhile, multiple secondary polymer templates can be replicated from the master mold and used in parallel, which is an effective way to increase the production throughput.

The desirable properties in plastic films to be used as nanoimprint templates are moderate mechanical strength, low surface energy, ultraviolet (UV) transparency, chemical stability, processing convenience, and flexibility particularly when used in continuous roll-to-roll UV-cured nanoimprint

${ }^{\text {a)} E l e c t r o n i c ~ m a i l: ~ l i w d @ h k u . h k ~}$ lithography (UV-NIL) process. ${ }^{7}$ Various polymeric materials have been used as nanoimprint template materials, such as polydimethylsiloxane, ${ }^{8}$ ethylene-tetrafluoroethylene, ${ }^{9}$ polyurethane acrylate, ${ }^{10}$ polyimides, ${ }^{11}$ polyvinyl alcohol, ${ }^{12}$ Teflon, ${ }^{13}$ and polyvinyl chloride. ${ }^{14}$ However, few of these materials possess a satisfactory combination of all the desirable properties, and there is still a demand for an ideal polymeric material that can be used as a superior alternative template material.

Cyclic olefin copolymer (COC) is a relatively new family of plastic materials but increasingly getting popular. ${ }^{15}$ Its unique combination of a number of promising properties such as low water absorption, good optical transparency in near UV range, low surface energy, high strength, and high chemical resistance to acids and alkalis has made it an ideal material for optical devices ${ }^{16}$ and microfluidic devices. ${ }^{17} \mathrm{COC}$ also possesses all the required properties for nanoimprint templates. As a thermoplastic material, COC films or plates can be readily molded from expensive silicon or nickel master molds through a thermal nanoimprint process, and used as low-cost secondary templates in large-volume manufacturing. $\mathrm{COC}$ has a relatively low surface energy, which facilitates mold separation even without special surface treatment. Its flexibility makes it suitable to be used for roll-to-roll templates, while its relatively high modulus and strong mechanical strength enable nanoscale features to be transferred with high fidelity.

A variety of fabrication approaches have been demonstrated on COC materials, including direct structuring methods (e.g., laser ablation ${ }^{18}$ and micromilling ${ }^{19}$ ) and replication techniques (e.g., hot embossing, ${ }^{20}$ injection molding, ${ }^{21}$ and $\mathrm{NIL}^{22}$ ). Yet, most of the reported replication methods so far were demonstrated with the fabrication of rather large structures (in range of micrometers) and many of these studies have mainly focused on device applications of the COC 
TABLE I. Properties of the COC film.

\begin{tabular}{lc}
\hline \hline Property & Value \\
\hline Film thickness $(\mu \mathrm{m})$ & 100 \\
Glass transition temperature $\left({ }^{\circ} \mathrm{C}\right)$ & 78 \\
Coefficient of linear thermal expansion $\left(\mathrm{K}^{-1}\right)$ & $0.7 \times 10^{-4}$ \\
Young's modulus $(\mathrm{MPa})$ & 2600 \\
Water Absorption @ $24 \mathrm{~h}$ immersion at $23^{\circ} \mathrm{C}(\%)$ & $<0.01$ \\
Water contact angle $(\mathrm{deg})$ & $93 \pm 1.5$ \\
\hline \hline
\end{tabular}

nanostructures ${ }^{22,23}$ or on optimizing the topography and surface properties of the master mold to enhance the morphology of the replicated COC nanostructures. ${ }^{24-26}$ Systematic experimental study on the effects of processing parameters on fabricating COC secondary templates with nanoscale features and demonstration of using the COC secondary templates in subsequent pattern transfer are needed to develop a wellcontrolled and reproducible fabrication procedure for using COC secondary templates in large-volume nanoimprint-based manufacturing of emerging nanodevices.

In this study, we first investigated the thermal nanoimprint process for replicating nanoscale structures into COC films. The effects of nanoimprint processing parameters on the mold cavity filling and pattern transfer fidelity are experimentally studied using grating structures of various sizes down to $70 \mathrm{~nm}$ half-pitch. The height and morphology of replicated gratings were analyzed by atomic force microscopy (AFM) and scanning electron microscopy (SEM) characterization, and optimized processing parameters were determined for high pattern transfer fidelity and short nanoimprint processing time. Then we used the nanostructured COC films as secondary nanoimprint templates and further transferred the gratings into photo-curable epoxy by a UV-NIL process. Moreover, we also demonstrated the transfer of metallic nanostructures from nanostructured COC templates to fiber facets for sensing applications. The precisely replicated nanostructures on UVcured epoxy and on fiber facets demonstrate the feasibility of using COC material for secondary NIL templates.

\section{EXPERIMENT}

COC films (Grade 8007) used in our fabrication were obtained from TOPAS Advanced Polymers. These COC films were fabricated by extrusion process and their major properties are summarized in Table I. The films were cut into $2 \times 2 \mathrm{~cm}$ pieces, cleaned by ultrasonication in acetone for $5 \mathrm{~min}$, and then dried by purging it with pressurized nitrogen gas. The contact angle of distilled water on the COC film [shown in Fig. 1(a)] was measured to be $93^{\circ}$ using an optical goniometer (Sindatek Instruments, China). Ultraviolet-visible spectrometry [shown in Fig. 1(b)] was performed by a UV-Vis spectrometer (Perkin Elmer Ltd., USA) and confirmed superior transmission of COC films over the whole near-UV to nearinfrared wavelength range.

Figure 2 shows the schematic of our complete fabrication processes. Initially, the gratings of different periods of 420 , 280 , and $140 \mathrm{~nm}$ are replicated from silicon master molds to COC films by thermal NIL. Then, fabricated gratings on COC films are used as templates for two different applications. In one application, the COC grating template is used as a secondary mold to imprint gratings into UV curable epoxy by a UV NIL process. In the second application, the COC grating is first coated with a thin gold layer and then the gold layer is detached and transferred onto optical fiber facets. Details of the experiments are presented in Secs. II A-II C.

\section{A. Thermal nanoimprint fabrication on COC films}

A home-built thermal nanoimprint setup was used to pattern the COC films with nanostructures. This setup consists of a manual hydraulic press (Specac Ltd., UK), a set of electrically heated platens with temperature controller (Specac Ltd., UK), and a water circulation chiller (Grant Instruments, UK).

During thermal NIL process, a silicon mold with a grating pattern was manually positioned onto the COC film placed at the center of the lower plate of the hydraulic press. Then, the plates were heated to the required temperature, and certain imprinting pressure was applied and held for $5 \mathrm{~min}$. The heated platens were then cooled down to the demolding temperature of $50^{\circ} \mathrm{C}$, and the COC film was peeled off from the silicon mold. The silicon molds used in our experiment have grating patterns with periods of 420,280 , and $140 \mathrm{~nm}$, and depths of 125,120 , and $70 \mathrm{~nm}$, respectively. All gratings have a $50 \%$ duty cycle. In all the experiments in the present work, silicon molds were not treated with any antisticking layer, since the low surface energy of imprinted COC films ensured a reliable mold separation after processing. The characterization of samples was performed using a Hitachi S-4800 scanning electron microscope and a Bruker MultiMode-8 atomic force microscope.
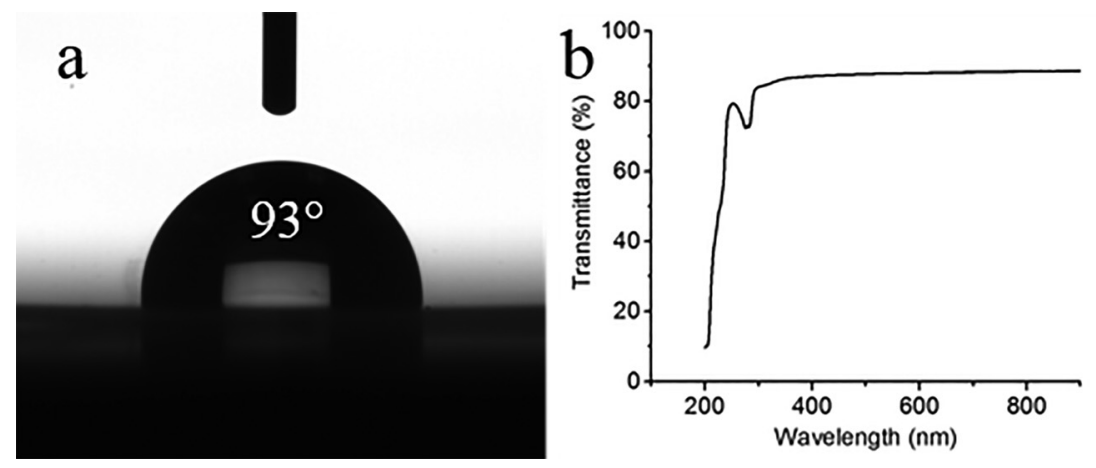

FIG. 1. Properties of the COC film: (a) water contact angle measurement and (b) optical transparency in UV and visible wavelength range. 


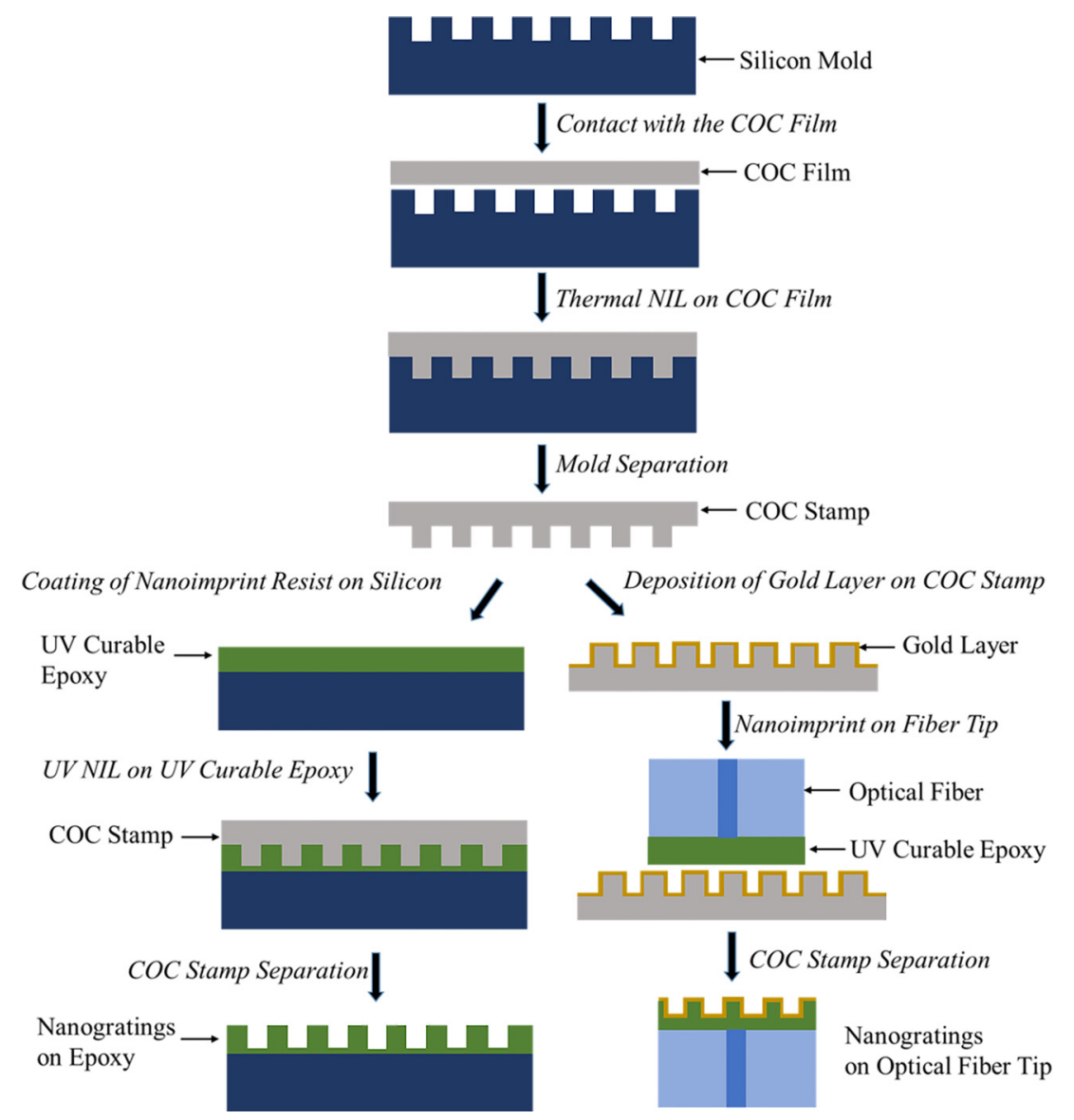

FIG. 2. (Color online) Schematic of the COC template fabrication and nanostructure transfer applications.

\section{B. UV nanoimprint using $\mathrm{COC}$ templates}

COC films with nanostructures fabricated by the aforementioned thermal nanoimprint process were used as secondary templates in subsequent UV nanoimprint lithography. These COC templates were not coated with an additional antisticking layer. In the UV nanoimprint, a thin layer of diluted NOA-61 UV-curable epoxy (Norland Products Inc., USA) was first spincoated on top of a silicon wafer and then the COC template was placed on top of it. Then, the template/substrate stack was exposed to a UV light-emitting diode (LED) light of $385 \mathrm{~nm}$ wavelength for 3 min with an applied pressure of approximately 20 bars to press the template and the substrate. The flexibility of the COC template ensured conformal contact and uniform applied pressure between the template and the substrate. The template/substrate stack was then removed from the imprint press after releasing the pressure. Finally, the COC template was manually peeled off from the cured epoxy, leaving nanostructures transferred on the epoxy for further device fabrication.

\section{Transferring metallic nanostructures onto optical fiber facets}

The COC templates can also be used for transferring functional metallic nanostructures onto nonconventional device substrates such as irregular or curved surfaces on which well-established lithography, etching, and deposition procedures cannot be readily deployed. Here, we demonstrate transferring metallic nanostructures onto optical fiber facets for plasmonic sensing applications. During the process, a thin gold layer was first deposited onto the COC template via e-beam evaporation and structured by the underlying pattern on the COC template. A multimode silica optical fiber (125 $\mu \mathrm{m}$ diameter) was prepared by stripping its jacket and cleaving into flat facet on one end. A uniform thin layer of UVcurable epoxy was transferred onto this fiber facet by first contacting the fiber facet on a layer of NOA-61 epoxy prespincoated on a flat silicon substrate and then separating the fiber from the silicon substrate, which is similar to the double-transfer nanoimprint process demonstrated elsewhere. $^{27}$ Then, the fiber facet, with the epoxy coated, was placed on top of the gold-covered COC template. After UV exposure, due to good adhesion between gold and cured NOA-61 epoxy, the gold structure adhered to the cured epoxy and was transferred from the COC template to the fiber facet. During this process, the optical fiber was fixed on a five-axis optical stage and the imprint process was monitored through a microscope. Therefore, conformal touching of the fiber facet on the template at specific locations can be achieved. 

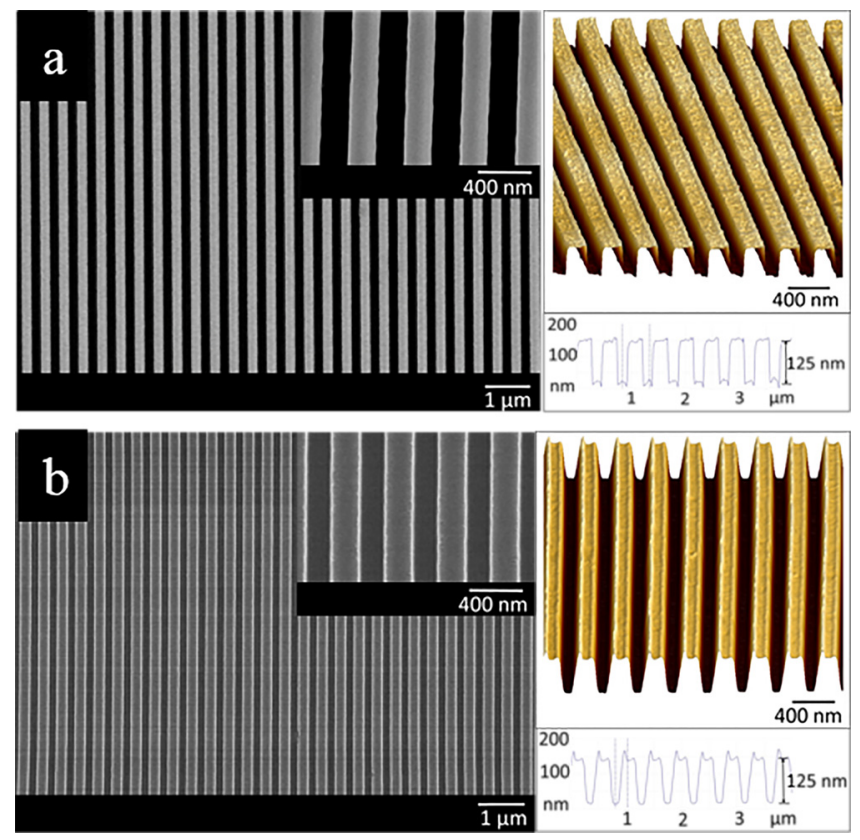

FIG. 3. (Color online) Fabrication results using nanogratings of $420 \mathrm{~nm}$ period, $210 \mathrm{~nm}$ line width, and $125 \mathrm{~nm}$ depth. (a) SEM and AFM image of original gratings on a silicon mold. (b) SEM and AFM image of the replicated gratings on COC (8007) film formed at imprint pressure $\mathrm{P}=6.2 \mathrm{MPa}$, imprint temperature $\mathrm{T}=100{ }^{\circ} \mathrm{C}$, and holding time $\mathrm{t}=5 \mathrm{~min}$.

\section{RESULTS AND DISCUSSION}

The SEM and AFM images of master silicon grating molds with periods of 420,280 , and $140 \mathrm{~nm}$ are presented in Figs. 3(a), 4(a), and 5(a), respectively. Similarly, the SEM images and AFM images of successfully replicated nanogratings with corresponding periods on COC films are shown in Figs. 3(b), 4(b), and 5(b), respectively. Each SEM image presents the gratings at two different magnifications while the corresponding AFM image shows the 3D profile and the cross-sectional height profile of the gratings. All the imprinted results shown in these images were fabricated using optimal imprinting parameters (imprinting pressure $\mathrm{P}=6.2 \mathrm{MPa}$, imprinting temperature $\mathrm{T}=100^{\circ} \mathrm{C}$ and holding time $\mathrm{t}=5 \mathrm{~min}$ ). Although the smallest grating line width used in this study was $70 \mathrm{~nm}$, we observed sub- $50 \mathrm{~nm}$ defect features replicated into the $\mathrm{COC}$ film from the master mold. It is clear from the presented images that the nanogratings in COC film are the complete copy of the nanogratings on COC master mold. However, by closely observing the AFM scans in Figs. 3(b) and 4(b), one can notice the "ears" on the edges of the gratings. These "ears" on the $420 \mathrm{~nm}$ period gratings and $280 \mathrm{~nm}$ period gratings are accurate replicates of impropriety in the $\mathrm{Si}$ master molds, which were caused by the over-etching of trench corners due to local crowding of electric field lines during reactive ion etching. Unlike the 420 and $280 \mathrm{~nm}$ period $\mathrm{Si}$ master molds, $140 \mathrm{~nm}$ period master mold does not have the excessively etched trench corner due to its narrower trench width, therefore its replicated gratings in COC film are without "ears."
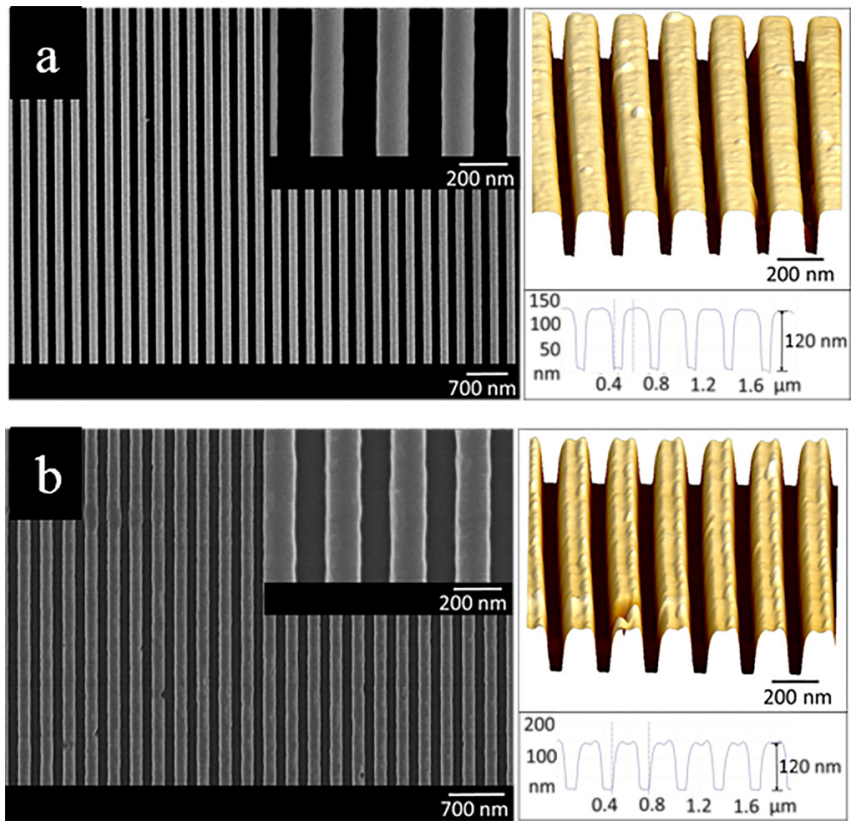

FIG. 4. (Color online) Fabrication results using nanogratings of $280 \mathrm{~nm}$ period, $140 \mathrm{~nm}$ line width, and $120 \mathrm{~nm}$ depth. (a) SEM and AFM image of original gratings on a silicon mold. (b) SEM and AFM image of the replicated gratings on COC (8007) film formed at imprint pressure $\mathrm{P}=6.2 \mathrm{MPa}$, imprint temperature $\mathrm{T}=100^{\circ} \mathrm{C}$, and holding time $\mathrm{t}=5 \mathrm{~min}$.

Beside these successful replications, a series of nanoimprint experiments were carried out on each grating mold using suboptimized imprinting parameters to (1) find the operating region (minimum pressure and minimum temperature required for perfect replication) for thermal NIL on the COC 8007 substrates used in this work, and (2) investigate the imprinted height as a function of imprinting parameters
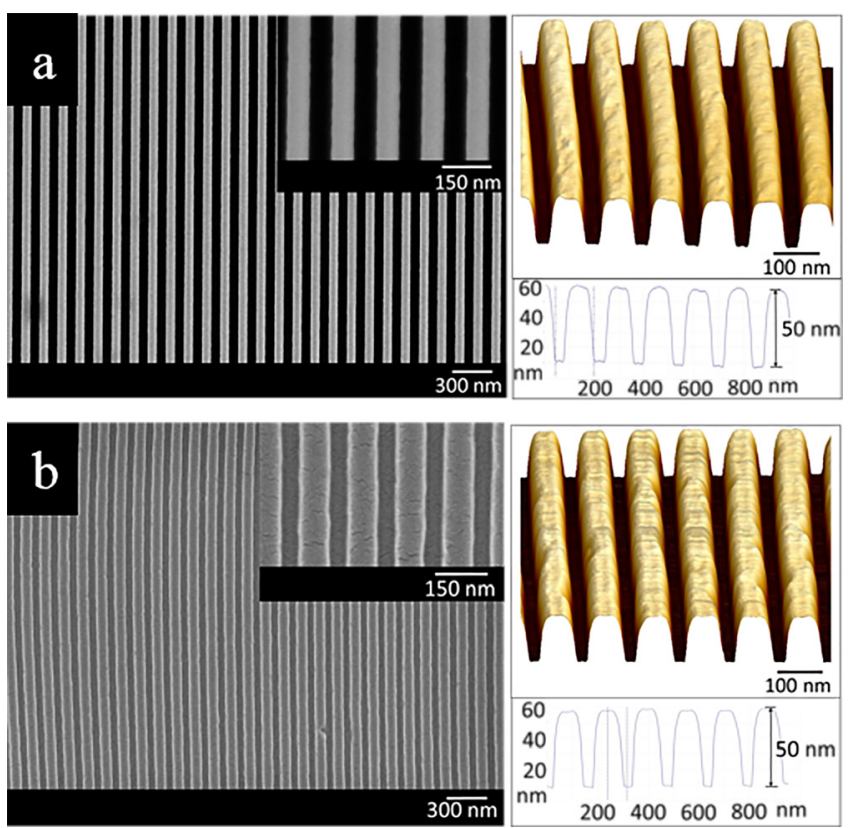

FIG. 5. (Color online) Fabrication results using nanogratings of $140 \mathrm{~nm}$ period, $70 \mathrm{~nm}$ line width, and $50 \mathrm{~nm}$ depth. (a) SEM and AFM image of original gratings on a silicon mold. (b) SEM and AFM image of the replicated gratings on COC (8007) film formed at imprint pressure $\mathrm{P}=6.2 \mathrm{MPa}$, imprint temperature $\mathrm{T}=100^{\circ} \mathrm{C}$, and holding time $\mathrm{t}=5 \mathrm{~min}$. 
during the thermal NIL process. For each set of experiments, the imprinted height was measured by AFM and compared to the depth of the trench on the silicon mold. Typical AFM images of partially filled gratings with periods of 420, 280, and $140 \mathrm{~nm}$ are sown in Figs. 6(a), 6(b), and 6(c), respectively. The summary of imprinting parameters for incomplete and complete replications of all three periods of nanogratings is shown in Fig. 7(a). It can be concluded from Fig. 6(a) that irrespective of grating size, the minimum imprinting pressure of $3 \mathrm{MPa}$ and imprinting temperature of $85^{\circ} \mathrm{C}$ is required for perfect replication through thermal NIL on COC 8007 substrates. However, it is clear from the quantitative data presented in Figs. 7(b), 7(c), and 7(d) that, under same suboptimal imprinting parameters, different height is replicated for each grating mold. For instance, at imprinting pressure of $12.55 \mathrm{MPa}$ and imprinting temperature of $80^{\circ} \mathrm{C}$, the replicated heights for 140,280 , and $420 \mathrm{~nm}$ period gratings are 30,70 , and $88 \mathrm{~nm}$, respectively. These incompletely replicated results indicate that the grating replication process is more influenced by imprinting temperature as compared to imprinting pressure. The reason for the phenomenon is that the modulus of the COC film abruptly changes near its glass transition temperature due to its viscoelastic behavior. At a temperature well below $\mathrm{T}_{\mathrm{g}}, \mathrm{COC}$ is in glassy region where its Young's modulus is high and remains constant over a wide range of temperature. The glassy $\mathrm{COC}$ film appears rigid and deforms in a more elastic manner. However, with increasing temperature over $\mathrm{T}_{\mathrm{g}}$, the thermal vibration becomes intense enough to overcome the potential barriers for rotation and translation of COC molecule segments. ${ }^{28}$ Therefore, the modulus of $\mathrm{COC}$ at the transition region decreases by several orders of magnitude, ${ }^{29}$ leading to a more pronounced effect of imprinting temperature over that of imprinting pressure.

When used for nanoimprint templates to replicate fine nanostructures, the template material is expected to have a high modulus, therefore minimum deformation during the imprint process. Figure 8 shows the AFM based measurement on the elastic modulus distribution of imprinted $420 \mathrm{~nm}$ period COC gratings together with the corresponding morphology image. The modulus mapping was obtained through the PeakForce Quantitative Nanomechanical Property Module on
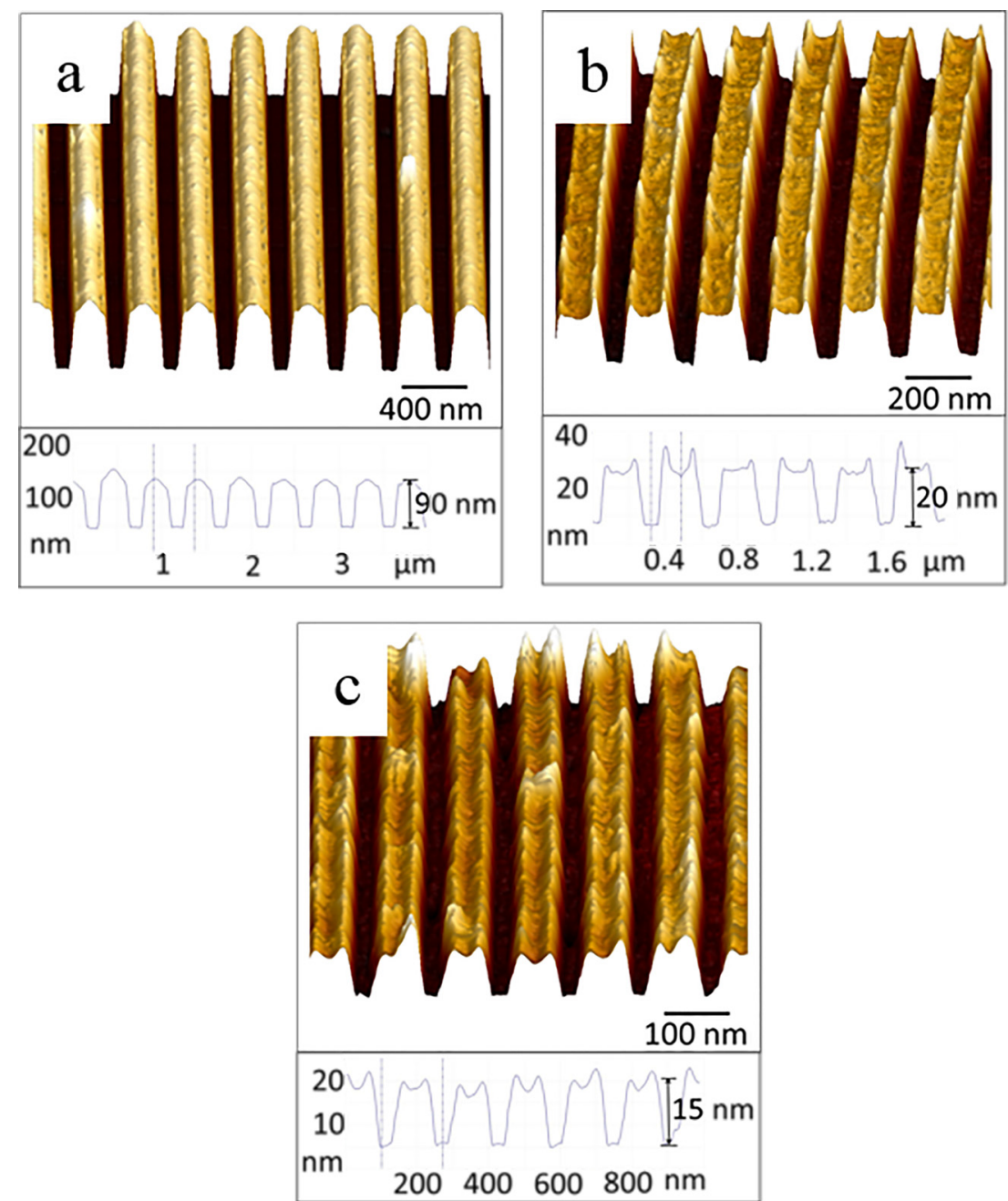

FIG. 6. (Color online) AFM images of typical COC gratings imprinted using suboptimal processing parameters: (a) Gratings having $420 \mathrm{~nm}$ period, $210 \mathrm{~nm}$ line width, and $90 \mathrm{~nm}$ depth; (b) gratings having $280 \mathrm{~nm}$ period, $140 \mathrm{~nm}$ line width, and $20 \mathrm{~nm}$ depth; and (c) gratings having $140 \mathrm{~nm}$ period, $70 \mathrm{~nm}$ line width, and $15 \mathrm{~nm}$ depth. 

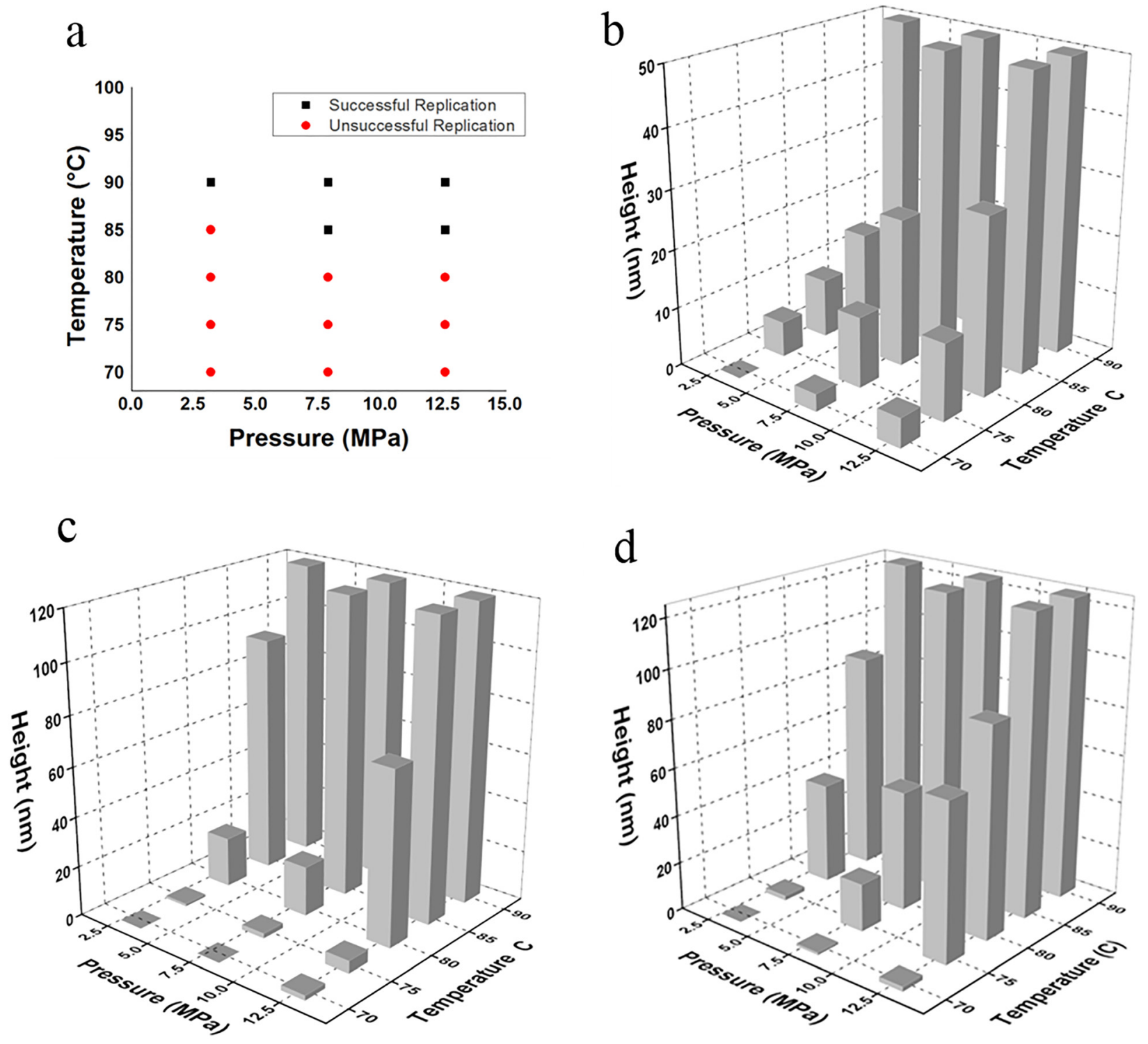

FIG. 7. (Color online) Influence of processing parameters on the pattern fidelity. (a) Summary of results for incomplete and complete replication of the gratings (irrespective of grating period). (b)-(d) Quantitative relationship between the depth of imprinted patterns, imprinting temperature, and imprinting pressure for $140 \mathrm{~nm}(50 \mathrm{~nm}$ height), $280 \mathrm{~nm}$ (120 nm height), and $420 \mathrm{~nm}$ (125 nm height) gratings, respectively. All grating molds have a 1:1 duty cycle.

the AFM using a Derjaguin Muller Toporov (DMT) model. ${ }^{30}$ The DMT elastic modulus measured at the center of the grating lines and at the center of the grating trenches ranges from 5 to $9 \mathrm{GPa}$, which is very high compared with other plastic materials used previously for nanoimprint templates. At the edge of grating lines, the AFM measurement cannot provide a reliable modulus value due to the effect of grating sidewalls. It is worth to note that the DMT modulus measured on the grating lines and in the trenches is apparently higher than the modulus measured on a featureless COC film, which is $\sim 2.7 \mathrm{GPa}$. This increase in the modulus presumably comes from the thermal NIL process where the film experiences a high compressive stress, which in turn increase the molecular density and hence the modulus of the film, ${ }^{31}$ but further study on this phenomenon and its effect on nanoimprint applications is needed.
The successfully fabricated nanogratings on COC film were used as a template to replicate nanogratings into UV curable epoxy by UV NIL process. In present work we used a readily available UV-curable epoxy, NOA-61, as the UV nanoimprint resist, although other UV-NIL resists can also be used. The SEM and AFM images of $140 \mathrm{~nm}$ period gratings transferred onto the epoxy layer are shown in Figs. 9(a) and 9(b), respectively. COC templates were tested for up to ten imprinting cycles and by investigating both the replicated grating profiles on the epoxy and the surface of the used template, we found no noticeable defects, surface deterioration, contamination, swelling, deformation, and collapse of features. This indicates that the $\mathrm{COC}$ templates are reasonably robust and durable. The well-replicated grating profiles on the epoxy for a large number of imprinting cycles demonstrate the feasibility of nanostructured COC films as low- 


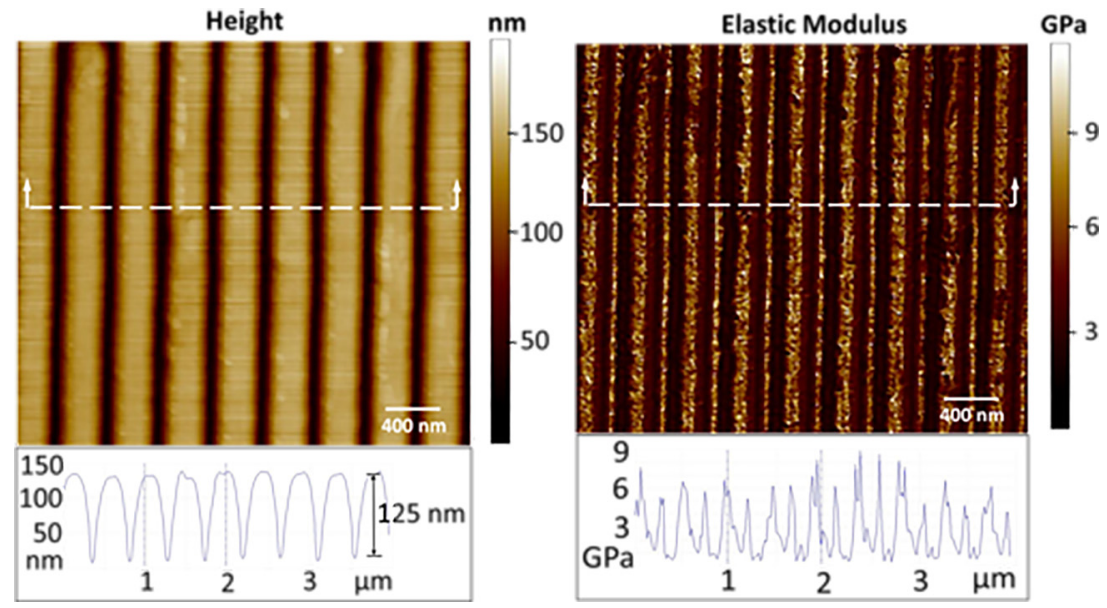

FIG. 8. (Color online) Representative topography and DMT modulus mappings of $420 \mathrm{~nm}$ period gratings COC film using PeakForce QNM imaging mode.

cost secondary NIL templates for transferring sub- $100 \mathrm{~nm}$ features. Moreover, these flexible templates can be utilized in roll-to-roll UV NIL process by wrapping it around an imprinting roller.

We also demonstrated transferring metallic nanostructures onto optical fiber facets using COC secondary templates. Optical fiber is a unique platform for various sensing applications ${ }^{32}$ for its microscopic cross-section and robust optical and mechanical properties. However, fabrication of integrated nanostructures on small fiber facets is quite difficult, compared with nanopatterning on conventional large-scale substrates. Some major challenges include coating of thin-film resist materials on small fiber facets, handling large-aspect-ratio optical fibers in processing tools, and so on. A number of approaches have been proposed to solve these issues, such as the double-transfer nanoimprint technique ${ }^{27}$ and transferring prefabricated nanostructures from a planar substrate. ${ }^{33}$ Because of its low cost, low surface energy and flexibility, we demonstrated that $\mathrm{COC}$ templates can be an ideal choice for transferring metallic nanostructures onto optical fiber facets. The processing sequence of transferring metallic structures from the COC secondary templates to fiber facets is depicted in Fig. 2. It is
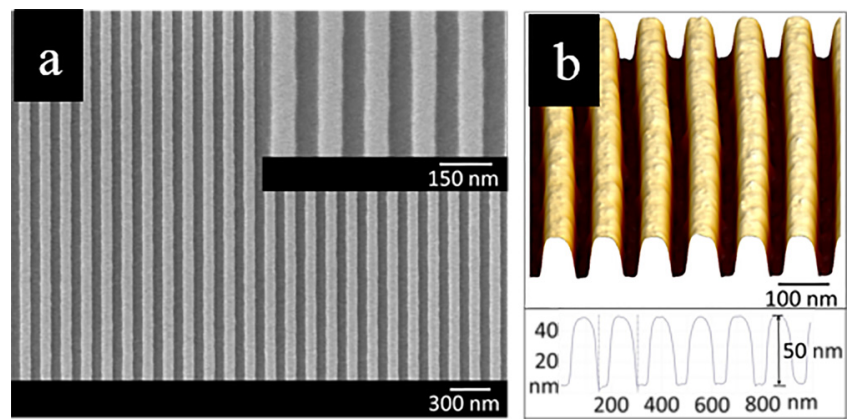

FIG. 9. (Color online) $140 \mathrm{~nm}$ period nanogratings on UV curable epoxy imprinted using the COC secondary template. (a) SEM images at two different magnifications. (b) The corresponding AFM image showing 3D profile and cross-sectional height profile. advantageous to use $\mathrm{COC}$ as the underlying substrate for the metal structure because the adhesion between them is typically weak. On the other hand, since the epoxy is also in partial contact with the underlying substrate during the metal transfer process, the weak adhesion between the COC substrate and the epoxy ensures an easy separation between the cured epoxy and the COC substrate. Figures 10(a) and 10(b) show the SEM and AFM images of successfully fabricated $420 \mathrm{~nm}$ period gold grating on $125 \mu \mathrm{m}$ diameter optical fiber facet using a fabrication procedure depicted in Fig. 2. The gold grating has a line width of $210 \mathrm{~nm}$ and a height of $125 \mathrm{~nm}$. These metallic nanostructures can be exploited to allow strong optical coupling of incident light to localized surface plasmons. With further design and optimization of the metallic structures to largely enhance local electrical field, such metallic nanostructures integrated with optical fibers can function as high-performance sensors for surface-enhanced spectroscopy techniques. ${ }^{34}$
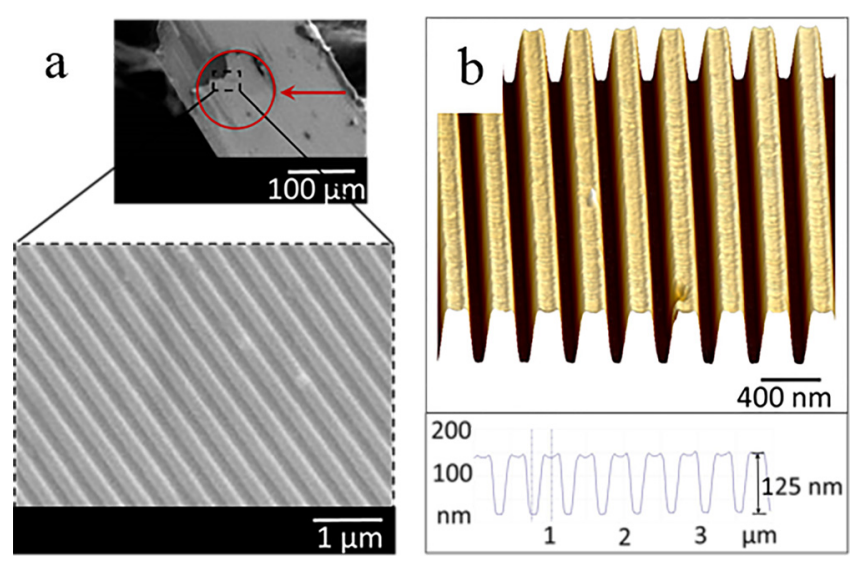

FIG. 10. (Color online) $420 \mathrm{~nm}$ period gold grating structures transferred onto fiber facet. (a) SEM images at different magnifications showing the optical fiber tip and the transferred gratings. Red arrow indicates the position of the optical fiber facet. (b) The corresponding AFM image showing 3D profile and cross-sectional height profile of the gratings. 


\section{CONCLUSIONS}

In summary, we experimentally investigate the feasibility of using thermal nanoimprint replicated COC secondary templates in patterning nanostructures through UV nanoimprint and transferring functional structures onto nonconventional substrates such as fiber facets. We first studied the processing parameters and replication fidelity of fabricating nanostructures on COC films through thermal nanoimprint. Nanogratings of different periods of 420,280, and $140 \mathrm{~nm}$ are successfully fabricated onto COC 8007 films using silicon molds with a minimum imprinting pressure of $3 \mathrm{MPa}$ and imprint temperature of $85^{\circ} \mathrm{C}$. It is concluded that during the imprinting, the influence of temperature on pattern fidelity is dominated as compared to that of applied pressure. As an application, the nanogratings are further transferred into UV-curable epoxy through a UV nanoimprint process using the nanostructured COC film as a template. The replicated structures on the UV-cured epoxy exhibit high fidelity compared with the COC templates and the original master molds. Moreover, we also used the fabricated $\mathrm{COC}$ gratings as a template to make and transfer metallic nanostructures onto optical fiber facets for fiber-based surface-enhanced sensing applications.

\section{ACKNOWLEDGMENTS}

This work was partially supported by the Young Scholar Program (61306123) of the National Natural Science Foundation of China and the Seed Funding Programme (201209160030) for Applied Research of the University of Hong Kong. The authors thank A. Barton and H. Han for providing COC samples, $\mathrm{Y}$. Chen and $\mathrm{H}$. Ge for discussion and help on some sample processing, and Nga Yu Hau, YuTing Huang, and S. P. Feng for contact angle and UV-Vis measurement.

${ }^{1}$ S. Y. Chou, P. R. Krauss, and P. J. Renstrom, Appl. Phys. Lett. 67, 3114 (1995).

${ }^{2}$ M. C. McAlpine, R. S. Friedman, and C. M. Lieber, Nano Lett. 3, 443 (2003).

${ }^{3}$ W. D. Li, F. Ding, J. Hu, and S. Y. Chou, Opt. Express 19, 3925 (2011).

${ }^{4}$ L. J. Guo, X. Cheng, and C. F. Chou, Nano Lett. 4, 69 (2004).
${ }^{5}$ D. Cheyns, K. Vasseur, C. Rolin, J. Genoe, J. Poortmans, and P. Heremans, Nanotechnology 19, 424016 (2008).

${ }^{6}$ S. H. Ahn and L. J. Guo, Adv. Mater. 20, 2044 (2008).

${ }^{7}$ H. Lan and H. Liu, J. Nanosci. Nanotechnol. 13, 3145 (2013).

${ }^{8}$ M. Bender, U. Plachetka, J. Ran, A. Fuchs, B. Vratzov, H. Kurz, T. Glinsner, and F. Lindner, J. Vac. Sci. Technol., B 22, 3229 (2004).

${ }^{9}$ S. H. Ahn and L. J. Guo, ACS Nano 3, 2304 (2009).

${ }^{10}$ S. M. Seo, T. I. Kim, and H. H. Lee, Microelectron. Eng. 84, 567 (2007).

${ }^{11}$ C. C. Wu, S. L. C. Hsu, and I. Lo, J. Nanosci. Nanotechnol. 10, 6446 (2010).

${ }^{12}$ C. D. Schaper, Nano Lett. 3, 1305 (2003).

${ }^{13}$ A. Workman, A. Marcia, C. P. Taussig, A. H. Jeans, and R. L. Cobene, J. Mater. Chem. 21, 14185 (2011).

${ }^{14}$ S. H. Hong, J. Y. Hwang, H. Lee, H. C. Lee, and K. W. Choi, Microelectron. Eng. 86, 295 (2009).

${ }^{15}$ H. Lee, S. Hong, K. Yang, and K. Choi, Appl. Phys. Lett. 88, 143112 (2006).

${ }^{16}$ P. I. Okagbare, J. M. Emory, P. Datta, J. Goettert, and S. A. Soper, Lab Chip 10, 66 (2010).

${ }^{17}$ P. S. Nunes, P. D. Ohlsson, O. Ordeig, and J. P. Kutter, Microfluid. Nanofluid. 9, 145 (2010).

${ }^{18}$ F. Bundgaard, G. Perozziello, and O. Geschke, Proc. Inst. Mech. Eng., Part C 220, 1625 (2006).

${ }^{19}$ J. Steigert et al., J. Micromech. Microeng. 17, 333 (2007).

${ }^{20}$ P. Leech, J. Micromech. Microeng. 19, 055008 (2009).

${ }^{21}$ V. Kalima, J. Pietarinen, S. Siitonen, J. Immonen, M. Suvanto, M. Kuittinen, K. Mönkkönen, and T. Pakkanen, Opt. Mater. 30, 285 (2007).

${ }^{22}$ L. Malic, B. Cui, M. Tabrizian, and T. Veres, Opt. Express 17, 20386 (2009).

${ }^{23}$ C. Vannahme, S. Klinkhammer, M. B. Christiansen, A. Kolew, A. Kristensen, U. Lemmer, and T. Mappes, Opt. Express 18, 24881 (2010).

${ }^{24}$ M. Matschuk and N. B. Larsen, J. Micromech. Microeng. 23, 025003 (2013).

${ }^{25}$ K. Mönkkönen, T. T. Pakkanen, J. Hietala, E. J. Pääkkönen, P. Pääkkönen, T. Jääskeläinen, and T. Kaikuranta, Polym. Eng. Sci. 42, 1600 (2002).

${ }^{26}$ N. Gadegaard, S. Mosler, and N. B. Larsen, Macromol. Mater. Eng. 288, 76 (2003).

${ }^{27}$ Y. Shen et al., Nanotechnology 24, 465304 (2013).

${ }^{28}$ M. T. Shaw and W. J. MacKnight, Introduction to Polymer Viscoelasticity (Wiley, New York, 2005).

${ }^{29}$ R. Jena, X. Chen, C. Yue, and Y. Lam, J. Micromech. Microeng. 21, 085029 (2011).

${ }^{30}$ B. V. Derjaguin, V. M. Muller, and Y. P. Toporov, J. Colloid Interface Sci. 53, 314 (1975).

${ }^{31}$ Y. S. Woo, J. K. Kim, D. E. Lee, K. Y. Suh, and W. I. Lee, Appl. Phys. Lett. 91, 253111 (2007).

${ }^{32}$ G. Kostovski, P. R. Stoddart, and A. Mitchell, Adv. Mater. 26, 3798 (2014).

${ }^{33}$ S. Scheerlinck, P. Dubruel, P. Bienstman, E. Schacht, D. Van Thourhout, and R. Baets, J. Lightwave Technol. 27, 1415 (2009).

${ }^{34}$ X. Yang, N. Ileri, C. C. Larson, T. C. Carlson, J. A. Britten, A. S. P. Chang, C. Gu, and T. C. Bond, Opt. Express 20, 24819 (2012). 